\title{
Reduced toxicity of liposomal nitrogen mustard prodrug formulation activated by an intracellular ROS feedback mechanism in hematological neoplasm models
} Mengting Lin ${ }^{1,2, \#}$, Wangwei Guo ${ }^{1, \#}$, Zhentao Zhang ${ }^{1, \#}$, Yi Zhou ${ }^{1}$, Jiejian Chen ${ }^{1,3}$, Tiantian Wang ${ }^{1}$, Xincheng Zhong ${ }^{1}$, Yiying $\mathrm{Lu}^{1}$, Qiyao Yang ${ }^{1,3}$, Qichun $\mathrm{Wei}^{3}$, Min $\mathrm{Han}^{1, *}$, Donghang $\mathrm{Xu}^{4,}$, Jianqing $\mathrm{GaO}^{1, *}$

${ }^{1}$ Institute of Pharmaceutics, College of Pharmaceutical Sciences, Zhejiang University, Hangzhou, 310058, China

${ }^{2}$ Hangzhou Institute of Innovative Medicine, College of Pharmaceutical Sciences, Zhejiang University, Hangzhou 310058, China

${ }^{3}$ Key Laboratory of Cancer Prevention and Intervention, the Second Affiliated Hospital, Zhejiang University, College of Medicine, Hangzhou, 310058, China

${ }^{4}$ Department of Pharmacy, the 2nd Affiliated Hospital, School of Medicine, Zhejiang University, Hangzhou, 310058, China

Corresponding author:

\section{Min Han}

Institute of Pharmaceutics, College of Pharmaceutical Sciences, Zhejiang University, Hangzhou 310058, People’s Republic of China, Tel +86-571-88208437, Email: hanmin@zju.edu.cn

\section{Donghang Xu}

Department of Pharmacy, the 2nd Affiliated Hospital, School of Medicine, Zhejiang University, Hangzhou, 310058, People's Republic of China, Tel +86-571-87783891, Email: xudonghang@zju.edu.cn

\section{Jianqing Gao}

Institute of Pharmaceutics, College of Pharmaceutical Sciences, Zhejiang University, Hangzhou 310058, People’s Republic of China, Tel +86-571-88208436, Email: gaojianqing@ zju.edu.cn

First authors

\#: These three authors contributed equally 


\section{Materials}

4-bromomethylphenylboronic acid pinamate and NM were purchased from Aladdin Co. Ltd. (Shanghai, China), and bisphosphoryl-phosphatidyl-Ethanolamine-polyethylene glycol 2000 (DSPE-PEG2000) and distearoyl-phosphatidyl-choline

(DSPC)

1 ,

2-dioleoyl-sn-glycero-3-phosphocholine (DOPC), 1, 2-dimyristoyl-sn-glycero-3-phosphocholine (DMPC), phosphor-lipidused S100 (Soybean Lecithin), hydrogenated soybean phospholipids (HSPC) were obtained from Avanti Polar Lipids (CA, USA). 3,3'-dioctadecyloxacarbocyanine perchlorate (DiO), 1,1'-dioctadecyl-3,3,3',3'-tetramethylindocarbocyanine perchlorate (DiI), Hoechst 33342 were obtained from Beyotime (Shanghai, China)

\section{Methods}

\section{NM degradation profiles}

To evaluate the NM degradation profiles, NM solutions $(10 \mathrm{mg})$ in a phosphate buffer saline (PBS) $(\mathrm{pH} 7.4,10 \mathrm{~mL})$ at $37^{\circ} \mathrm{C}$. At various time intervals, aliquot of the solution $(500 \mu \mathrm{L})$ was withdrawn and replaced with the same volume of fresh media. Solutions were extracted with cyclohexane-ethyl acetate (1:1, V/V) and followed by GC-MASS analysis.

\section{Cell targeting analysis}

Five phospholipids (DSPC, DOPC, DMPC, HSPC and Soybean Lecithin), cholesterol, DSPE-PEG2000 (5:1:0.8, w:w:w) and Hoechst solution $(80 \mu \mathrm{g} / \mathrm{mL})$ were dissolved in $15 \mathrm{~mL}$ chloroform and then the mixture were emulsified for $8 \mathrm{~min}$ to from a white emulsion. The solvent was removed through rotary evaporation to constract an adherent clear gel. The solution was added $10 \mathrm{~mL}$ PBS and evaporated until the gel dissolved with a bluish opalescence were facilitate through sonication for 3 min under the ice bath. A uniformly dispersed Hoechst liposome, and dialyzed (MW: 8000-14000) overnight to remove the free hoechst solution.

In order to establish a circulation tumor model, we constructed a peristaltic pump device, and investig ated its power output and cycle time. The power output and cycle time were optimized for subsequent experiment of cell-targeted uptake.

\section{Results}


N, N-bis(2-chloroethyl)methyl ammonium hydrochloride

4-(bromomethyl)phenylboronic acid pinacol ester

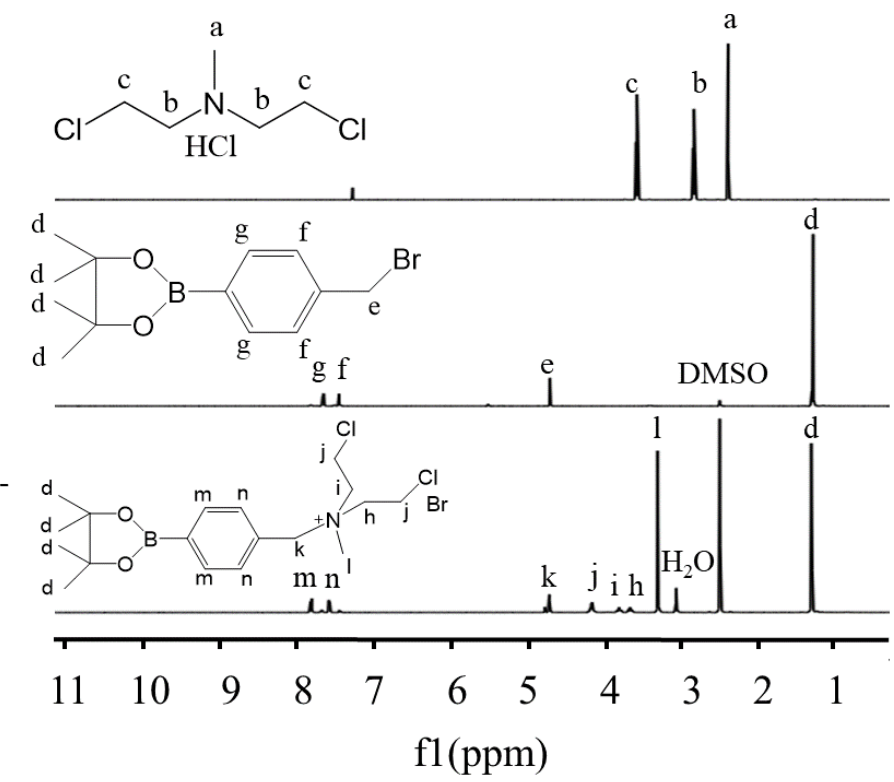

Figure S1 ${ }^{1} \mathrm{H}$ NMR spectra of N,N-bis(2-chloroethyl)methyl ammonium hydrochloride, 4-(bromomethyl) phenylboronic acid pinacol

ester

and

N,N-bis(2-chloroethyl)-N-methy l-N-[4-(4,4,5,5-tetramethy 1-1,3,2-dioxaborolan-2-yl)phenyl]

methanaminium bromide (NM-Pro).

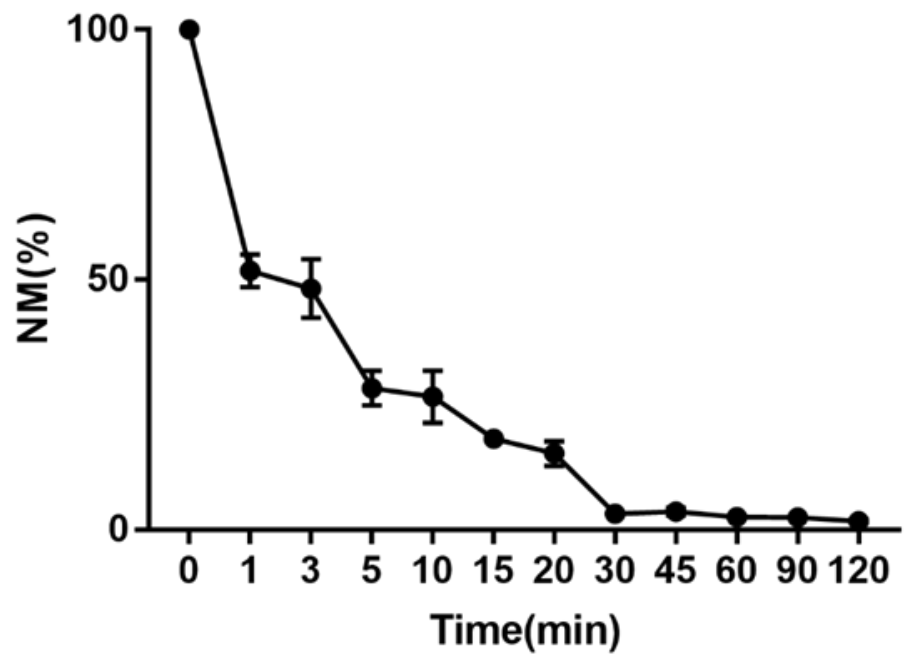

Figure S2 Degradation of NM (1.0 mg/mL) in PBS (pH 7.4, $\left.10 \mathrm{~mL}, 37^{\circ} \mathrm{C}\right)$. Solutions were extracted with cyclohexane-ethyl acetate $(1: 1, \mathrm{~V} / \mathrm{V})$ and followed by a GC-MASS analy sis. 

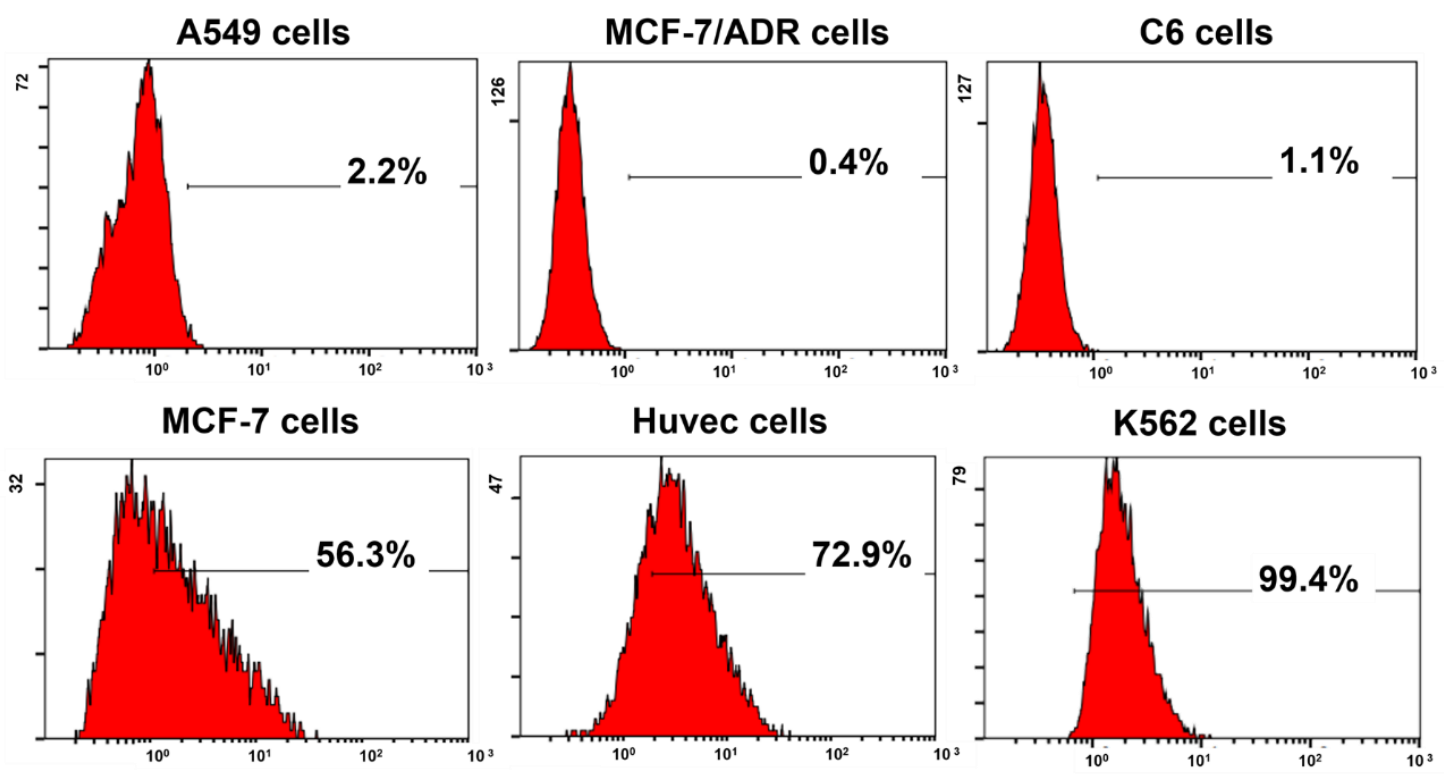

Figure S3 The intracellular ROS levels of the various cells were analyzed by incubating MCF-7, MCF7/ADR, A549, HUVEC, C6 and K562 cells with the formulations for $24 \mathrm{~h}$ at $37^{\circ} \mathrm{C}$. The fluorescence intensity at $488 \mathrm{~nm}$ was quantitatively measured using flow cytometry.
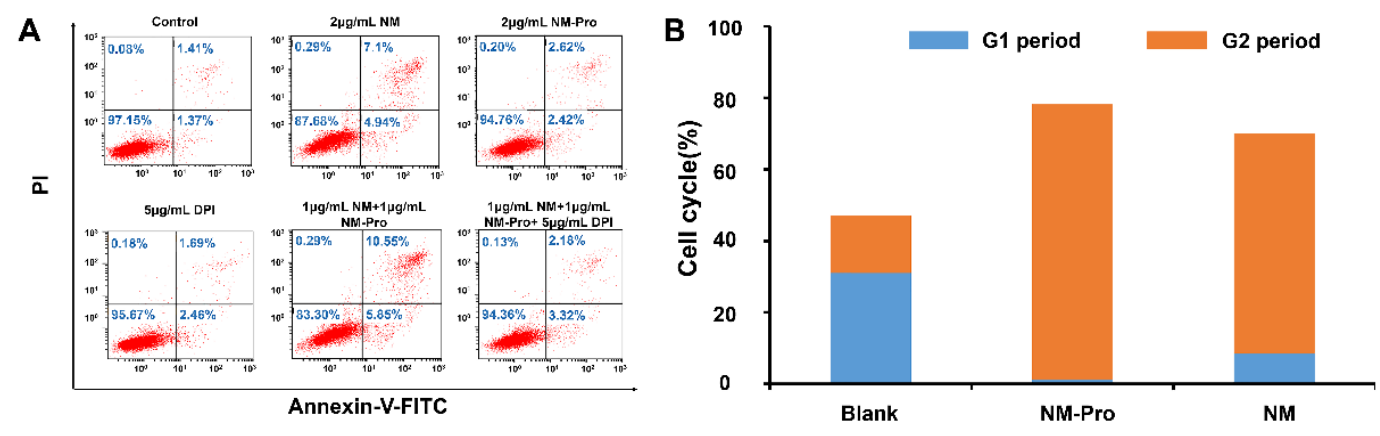

Figure S4. The K562 cell apoptosis and cell cycle assays. (A) Flow cytometry detected the effects of treatment with the combination of NM and NM-Pro on K562 cell apoptosis for 48 h. (B) Arrest of the K562 cell cycle by treatment with the combination of NM $(1 \mu \mathrm{g} / \mathrm{mL})$ and NM-Pro $(10 \mu \mathrm{g} / \mathrm{mL})$ for $48 \mathrm{~h}$. 


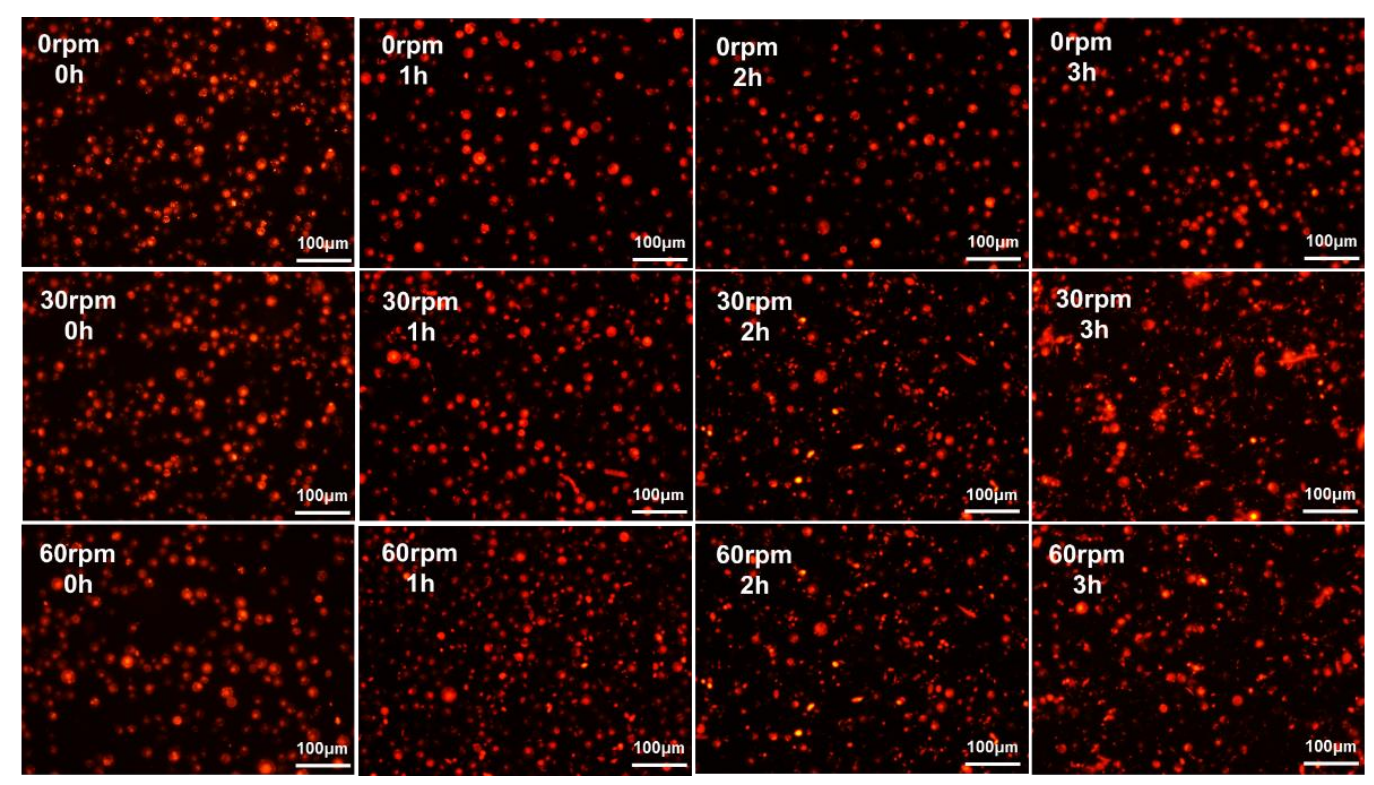

Figure S5 K562 cell state diagram after an incubation in the flow apparatus at different rotational speeds and cycle times of the peristaltic pump (red: DiI).

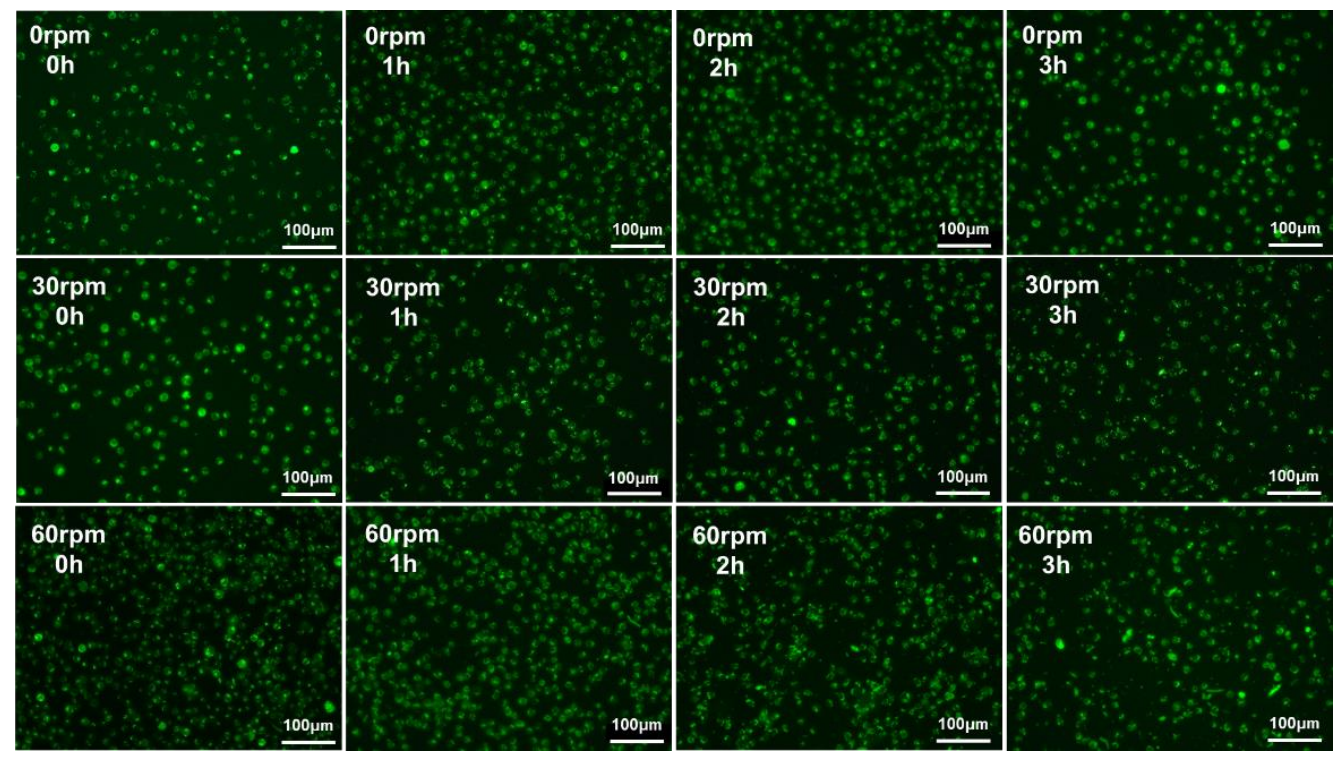

Figure S6 THP-1 cell state diagram after an incubation in the flow apparatus at different rotational speeds and cycle times of the peristaltic pump (green: DiO). 

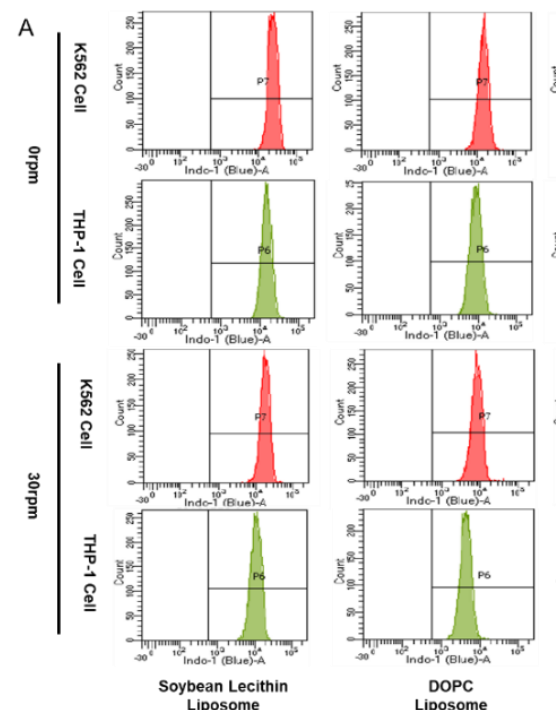

DOPC
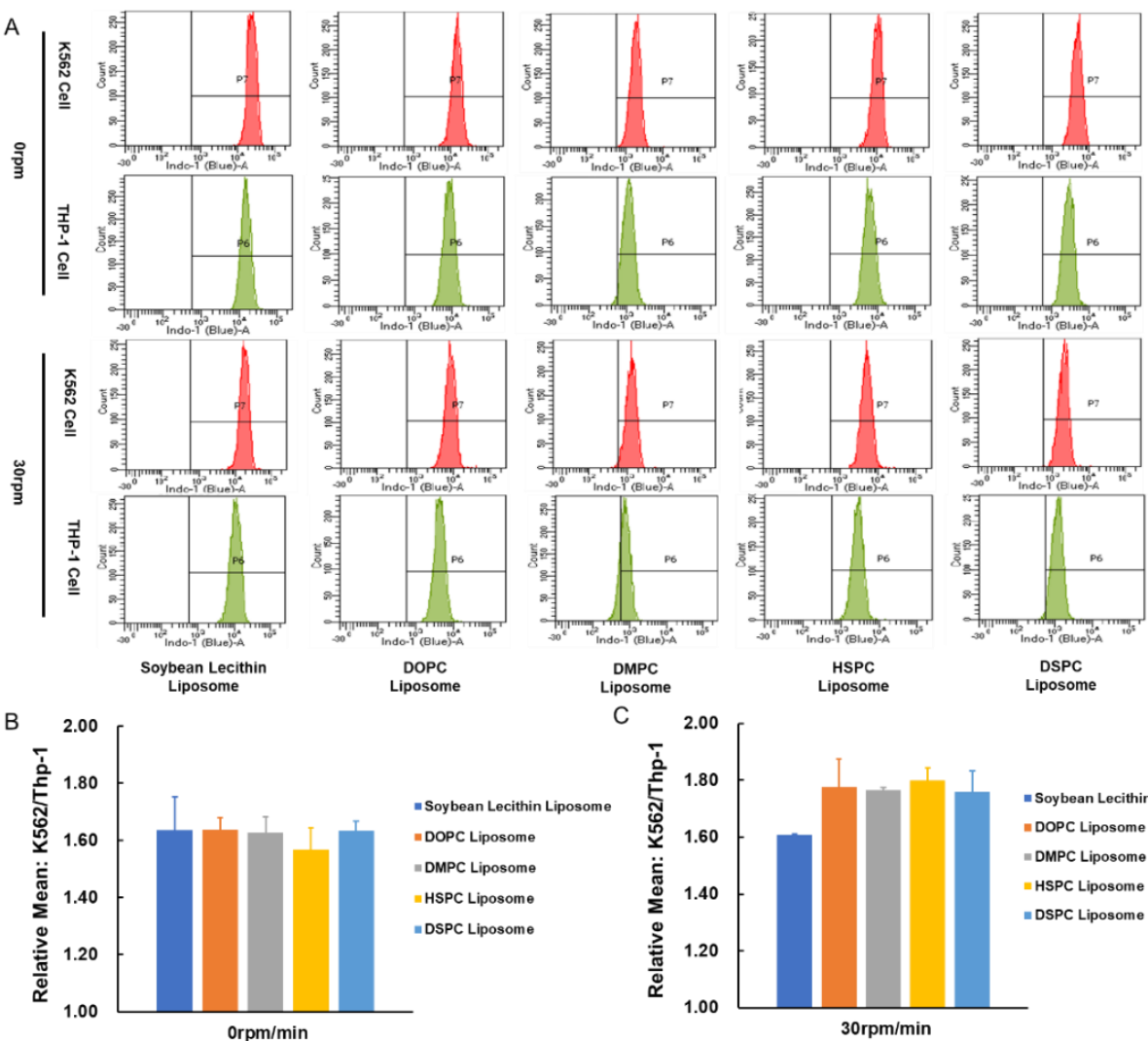

C

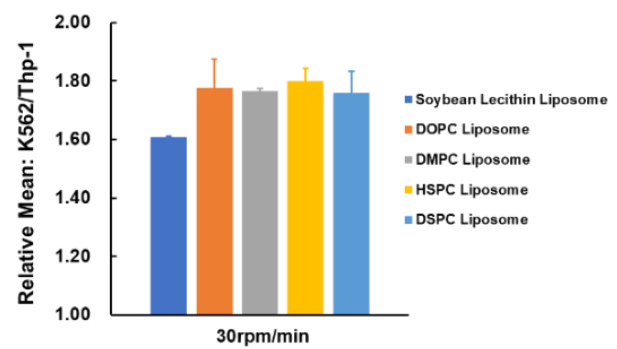

Figure S7 Multicolor flow cy tometry was performed to investigate the targeting of liposomes to K562 and THP-1 cells. A: Soybean lecithin liposomes, DOPC liposomes, DMPC liposomes, HSPC liposomes and DSPC liposomes targeting K652 (red) and THP-1 (green) cells after an incubation in the flow apparatus at different rotational speeds and cycle times of the peristaltic pump. B: Relative mean liposome uptake by K562 and THP-1 cells at rest in the peristaltic pump. C: Relative mean liposome uptake by K562 and THP-1 cells at $30 \mathrm{rpm}$ in peristaltic pump.
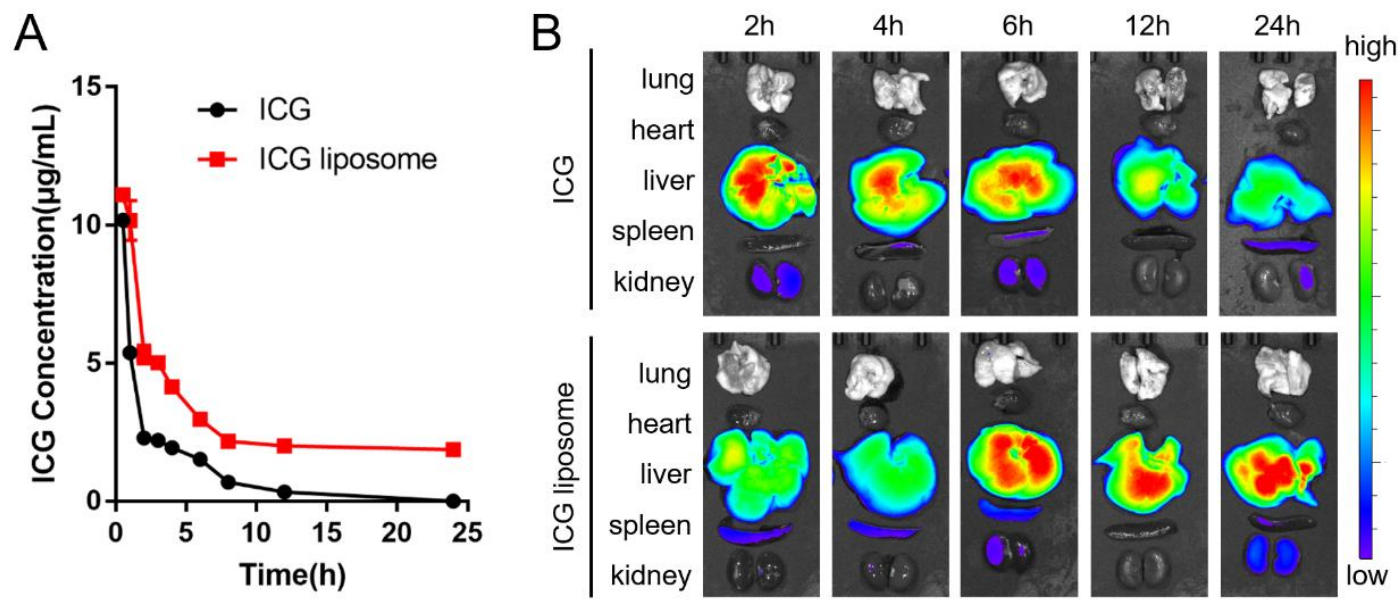

Figure S8. Pharmacokinetics and elimination of ICG-liposomes and the ICG solution. (A) Blood concentration-time curves of free ICG and ICG liposomes in SD rats an after intravenous injection (ICG dose, 1.5 
$\mathrm{mg} / \mathrm{kg}$ ), $\mathrm{n}=3$. (B) Elimination of ICG at 2, 4, 6, 12, and $24 \mathrm{~h}$ after the intravenous injection of various formulations $(1.5 \mathrm{mg} / \mathrm{kg}$ equivalent $\mathrm{ICG})$ in $\mathrm{SD}$ rats.

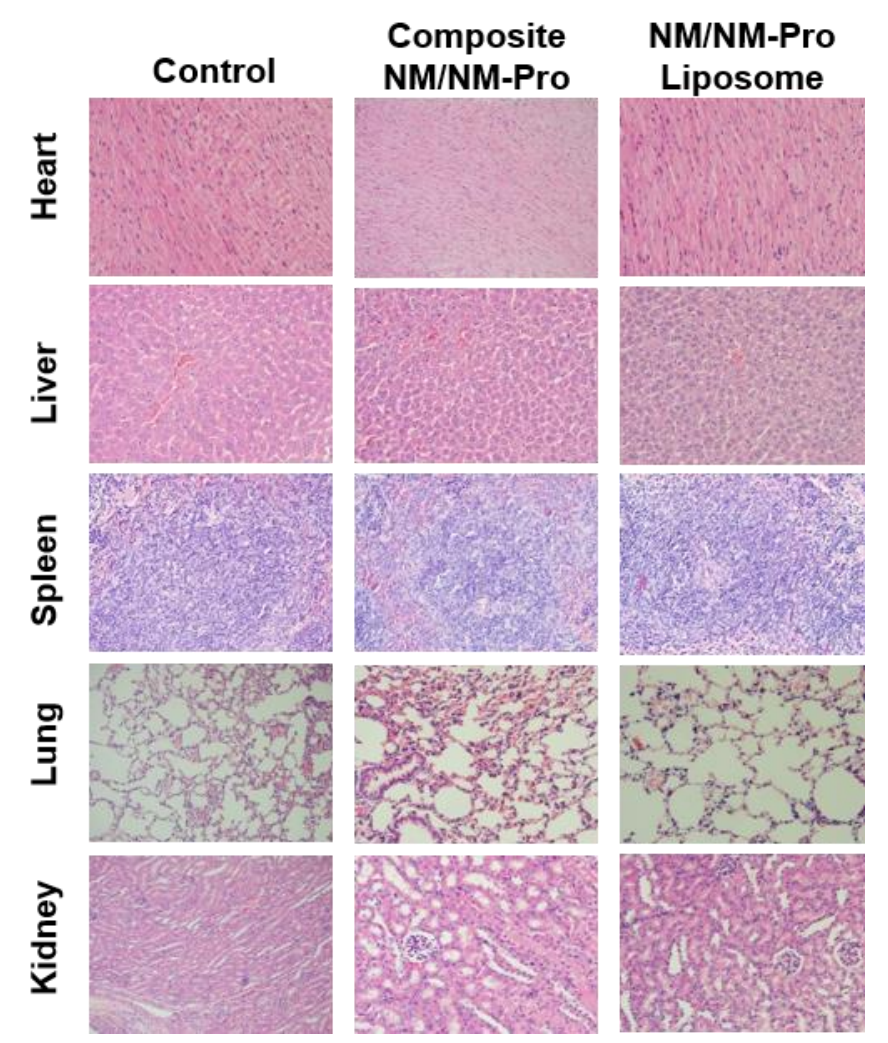

Figure S9 Images of H\&E staining in mouse organs (heart, liver, spleen, lung, and kidney). 


\section{References:}

1. Kuang, Y.; Balakrishnan, K; Gandhi, V.; Peng, X. Hydrogen Peroxide Inducible DNA Cross-Linking Agents: Targeted Anticancer Prodrugs. Journal of the American Chemical Society 2011, 133, (48), 19278-19281. 